\title{
Microelectrode Recording Equipment for Movement Disorders
}

a report by

Alpha 0 mega
Microelectrode recording (MER) began in the research laboratory as a tool for scientists to record the activity of individual neurons in the brain. The process was eventually introduced into a clinical setting and, in the 1960s, MER became an important device for target localisation in lesioning surgeries, such as pallidotomy and thalamotomy, used in the treatment of movement disorders. The subsequent introduction of levadopa decreased the popularity of surgical intervention for movement disorders until its rebirth in the 1980s. Currently, electrophysiological monitoring helps neurosurgical teams to accurately isolate specific neurological cells targeted for deep brain stimulator (DBS) implantation, in addition to the lesioning procedures. MER is a critical tool in the effective treatment of disorders such as Parkinson's disease (PD), dystonia and essential tremor, among others.

Initially, MER devices were home-built systems pieced together from various components. The compoents required for a fully functioning MER setup include:

- a microelectrode or semi-microelectrode;

- amplification and filtering;

- oscilloscope or video monitor; and

- audio monitor and microdrive ${ }^{1}$.

In the past these set-ups were compiled by researchers or technicians and provided clumsy, inadequate solutions that made the process of MER cumbersome and complicated. In recent years, many commercially available systems have emerged in an effort to provide elegant solutions for researchers and movement disorder specialists. Only a few of these devices have successfully stood the test of time or satisfied the rigorous standards of the Food and Drug Administration (FDA) in the US

One system that stands out from the rest is the MicroGuide Pro ${ }^{\mathrm{TM}}$ by Alpha Omega Engineering
(Nazareth, Israel). For many years, Alpha Omega successfully developed state-of-the-art MER equipment for movement disorder research, so transitioning to the clinical setting was the logical next step. By working in close collaboration with scientists and neurosurgeons from around the world, the experts at Alpha Omega took its existing MER technology and adapted it to create a single fully integrated system called the MicroGuide Pro $^{\mathrm{TM}}$. The first version was released for clinical use in 1999. Currently, the MicroGuide Pro ${ }^{\text {TM }}$ is the most comprehensive MER guidance system available for functional neurosurgery.

Many features set the MicroGuide Pro ${ }^{\text {TM }}$ apart from other commercially available systems. One feature that is a favourite among neurosurgeons is the integrated display screen that provides access to all electrophysiological data and system functions on one screen. These functions can be conveniently controlled from anywhere in the operating room using the hand-held control pad. Another favoured feature is the integrated impedance checker that allows the user to monitor the integrity and quality of the microelectrodes throughout the surgery. For those who conduct clinical research, the MicroGuide Pro ${ }^{\mathrm{TM}}$ offers exceptional data logging and analysis capabilities. Throughout the surgery, the trajectory is tracked and can be annotated to mark significant events. After the surgery, the trajectory map can be printed from the printer included with system, and all of the data will be saved to a disk for later review.

In addition to all of these functions, there are two elements of the MicroGuide Pro $^{\text {TM }}$ that are unparalleled in other commercially available systems. The first feature is a multi-channel recording that allows the user to record simultaneously from up to five electrodes. The ability to record from multiple electrodes increases the efficiency and quality of an electrode pass and reduces time in the operating room. The second

1. Slavin K, Holsapple J, "Microelectrode Techniques: Equipment, Components, and Systems", in: Israel Z, Burchiel K, eds. Microelectrode Recording in Movement Disorder Surgery, New York: Thieme Medical Publishers; (2004): pp. 14-22. 
Figure I: MicroGuide Pro ${ }^{\mathrm{TM}}$ in the Operating Room

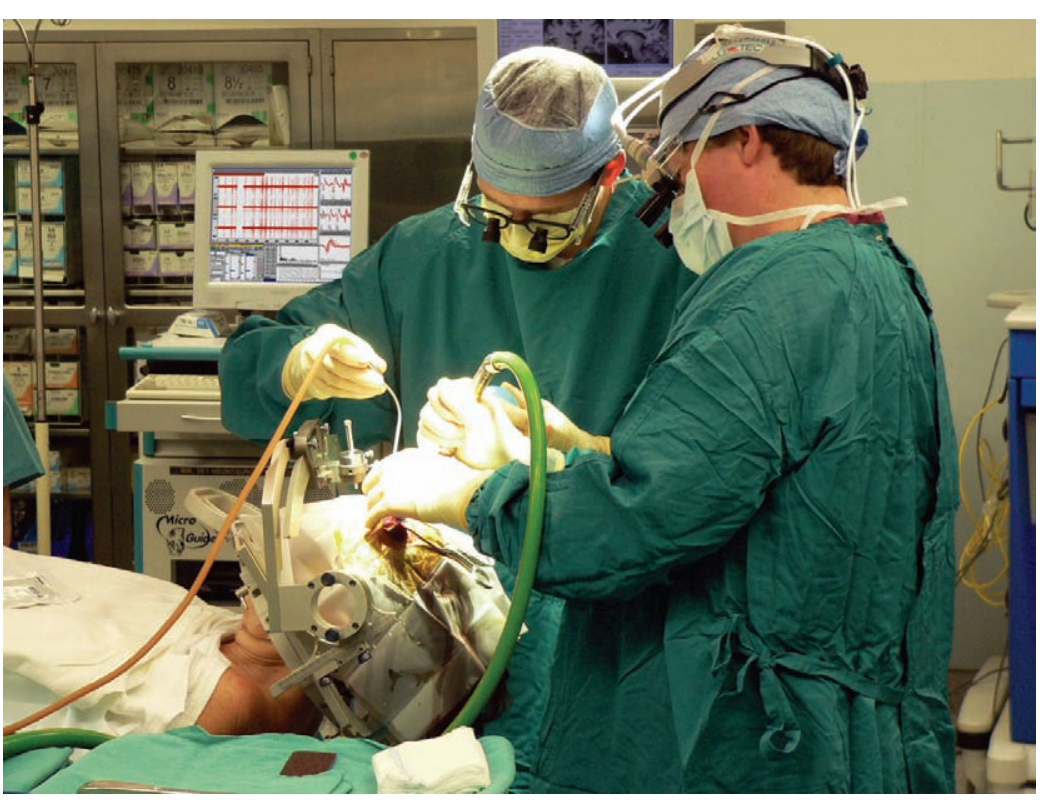

Figure 2: MicroGuide Pro ${ }^{\mathrm{TM}}$

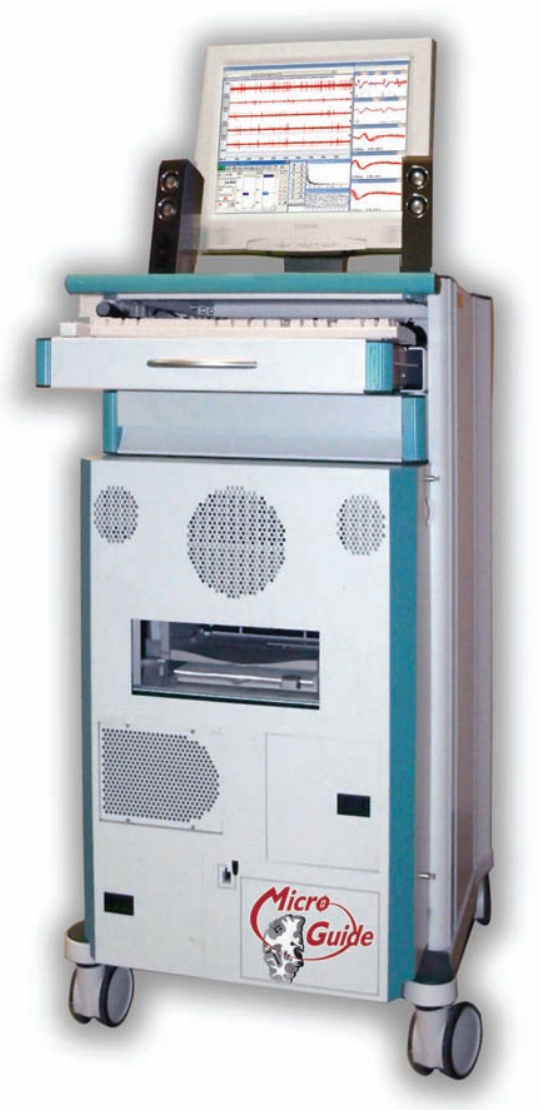

feature is integrated microstimulation, which can be used to verify the optimal target location has been reached. In order to use microstimulation with other systems, the neurosurgical team must utilise an entirely separate device, which exhausts valuable operating room (OR) time. According to Slavin and Burchiel,
"The MicroGuide system seems to be the most complete system on the market at present for multichannel recording and integrated micro-stimulation. It's easy to use and the manufacturer provides excellent customer service and technical support." 2

In another article, by Slavin and Holsapple, they reaffirm that,

"The MicroGuide system is probably the best option for those who consider doing serious processing of microrecording data and prefer to use multiple electrodes for deep subcortical exploration." 1

A main component of any MER system is the microdrive, the tool that physically advances the electrodes into the brain. Many of the microdrives available on the market are difficult to assemble and too heavy, creating torque on the frame. The NeuroDrive ${ }^{\mathrm{TM}}$, developed by Alpha Omega in 2005, was designed to alleviate some of the hardships associated with the microdrives of the past. The NeuroDrive is significantly smaller than previous surgical microdrives, and it comes preassembled, therefore minimising preparation time for surgery. During the procedure, the user can continue recording while the electrodes are in motion because the motor of the NeuroDrive is practically silent. A further feature unique to microdrives from Alpha Omega is the optional BenGun which is used for the simultaneous advancement of five electrodes. The BenGun is an multi-electrode holder that guides the canulae in an ' $\mathrm{X}$ ' shape and "has proved to be particularly useful for mapping the subthalamic nucleus, based on the experience of Benabid et al., in Grenoble, France"2

With advanced technology like the NeuroDrive and MicroGuide Pro ${ }^{\mathrm{TM}}$, functional neurosurgery procedures are gradually becoming standardised. The reliability of the MicroGuide Pro ${ }^{\mathrm{TM}}$ system allows the surgeon to develop a methodology for MER. Undoubtedly with each patient and in each case the neurosurgical team will encounter some variables; however, the MicroGuide Pro $^{\text {TM }}$ "incorporates all tools and feedback necessary to ensure a successful surgery", according to the President and founder of Alpha Omega, Imad Younis.

Although the necessity of MER during surgery has long been debated, the benefits cannot be denied. Due to the nature of the surgery, actual physical visualisation of the target is not possible, therefore some kind of intra-operative physiological

2. Slavin KV and Burchiel KJ, "Instrumentation, Technique, and Technology: MicroGuide Microelectrode Recording System”, Neurosurgery (2002);51 (number 1): pp. 276-278. 
targeting is required. Pre-operative planning is necessary to forecast the placement of the electrode lead; however, an article published in 2005 by the Korean Neurosurgical Society, Kim et al., suggested that,

"A number of factors limit the precision of anatomic targeting based upon historical image sets. These factors include; (1) image distortion of magnetic resonance imaging (MRI); (2) imperfect visualisation of the target structure that precludes the ability to compensate for anatomic variability in an individual; (3) brain shift that occurs after pre-operative imaging is complete; and (4) a specific physiological function may not always coincide with the same anatomic location." 3

Using MER, the specialist can correct the initial target during the surgery thereby increasing the efficiency of positioning. ${ }^{3}$ In addition, Dr Ronald Tasker of Toronto, Canada, said,

"The objectivity of recording compensates for any deficit of engagement on the part of the patient during microstimulation while the availability of both recording and stimulation data from the same site is a very powerful localising methodology." 4

Furthermore, he states,

"Every stereotactic functional procedure is, in fact, a neurophysiological experiment in which microelectrodes allow elegant studies of normal and abnormal brain function, hopefully increasing our understanding of the problems we are treating and leading to better management." 4

Future

Alpha Omega is working to define the future of MER, therefore the company redirects a majority of its funding back into research and development (R\&D). One device that is still in development is a smaller, more elegant version of the MicroGuide Pro $^{\mathrm{TM}}$. This future device will be portable and will reduce the whole system down to a size similar to a personal digital assistant (PDA). The solution will be
Figure 3: Neuro Drive

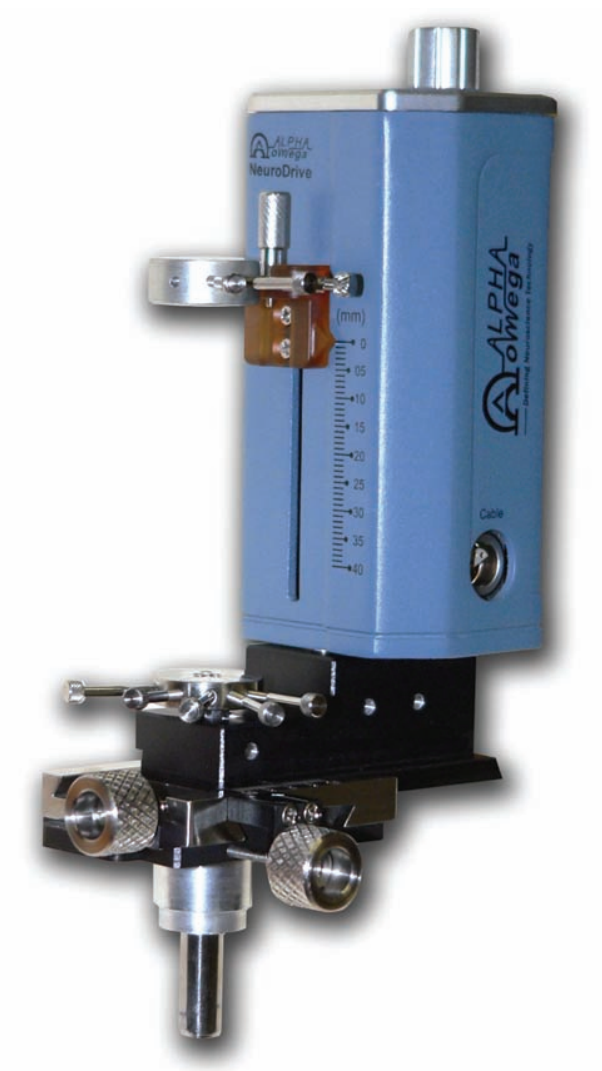

perfect for neurophysiologists and neuro-surgeons who work at multiple hospitals and need a portable solution. According to Imad Younis, the next generation of device will be a simplified system that will use "state-of-the-art processing technology."

In future MER systems, the target will be located automatically by implementing various algorithms. This revolutionary feature will substantially minimise surgical time and streamline the procedure. Automation will increase accuracy in target localisation while reducing the risks to the patient.

As deep brain stimulation (DBS) expands into new areas of the brain, or new applications, the technology required to implement safe and effective surgeries is evolving with it. The future of MER is promising with dedicated companies like Alpha Omega leading the way.

3. Kim S M, Hwang H S and De Salles A, "Shift of the Brain during Functional Neurosurgery" Journal of Korean Neurosurgical Society (2005);38(5): 359-364.

4. Tasker R, In: Israel Z, Burchiel K, eds. Microelectrode Recording in Movement Disorder Surgery, New York: Thieme Medical Publishers; (2004): vii. 